\title{
Evaluación de metodologías para recuento de garrapatas en fase de vida parásita
}

\author{
Evaluation of methodologies for counting of ticks in the \\ parasitic life phase
}

María Inés Rodríguez ${ }^{1,4}$; Roberto Martínez²; Lorena Núñez ${ }^{3}$

\section{Resumen}

\begin{abstract}
El estudio tuvo como objetivo comparar la exactitud de las pruebas o métodos de recuento de garrapatas ingurgitadas en hembras bovinas criadas bajo sistemas de producción extensiva. Se utilizaron 49 vacas adultas de los genotipos Criollo Ñeembucú, Pardo Suizo, Brangus y Nelore durante el estío en marzo de 2019. Se revisaron varias regiones corporales de manera secuencial mediante el método visual indirecto, palpatorio y finalmente el método directo, definido este último como patrón de referencia o gold standard. Para la estimación de los indicadores de validez y fiabilidad, comparación del comportamiento de las curvas de características operacionales del receptor (ROC) y pruebas de concordancia se recurrió al programa para análisis epidemiológico de datos tabulados Epidat 3.1. La sensibilidad y el valor predictivo negativo fueron marcadamente superiores para la prueba palpatoria. El deficiente valor del índice Kappa reveló que el acuerdo entre el método visual indirecto y directo fue debido al azar $(\mathrm{p}=0.2097)$. En el método palpatorio, la curva ROC mostró un área mayor en comparación con el método
\end{abstract}

${ }^{1}$ Departamento de Ciencias Morfológicas, Facultad de Ciencias Veterinarias, Universidad Nacional de Asunción, Paraguay

${ }^{2}$ Dirección General de Investigación Científica y Tecnológica, Universidad Nacional de Asunción, Paraguay

${ }^{3}$ Laboratorio de Diagnóstico de Enfermedades de los Animales Domésticos, Facultad de Ciencias Veterinarias, Universidad Nacional de Asunción, Paraguay

${ }^{4}$ E-mail: mrodriguez@vet.una.py; https://orcid.org/0000-0001-9278-7928

Recibido: 19 de septiembre de 2020

Aceptado para publicación: 10 de abril de 2021

Publicado: 23 de junio de 2021

CLos autores. Este artículo es publicado por la Rev Inv Vet Perú de la Facultad de Medicina Veterinaria, Universidad Nacional Mayor de San Marcos. Este es un artículo de acceso abierto, distribuido bajo los términos de la licencia Creative Commons Atribución 4.0 Internacional (CC BY 4.0) [https:// creativecommons.org/licenses/by/4.0/deed.es] que permite el uso, distribución y reproducción en cualquier medio, siempre que la obra original sea debidamente citada de su fuente original 
visual indirecto. Los resultados de la prueba de homogeneidad de las áreas ROC revelaron que la diferencia en cuanto a la eficacia de ambas pruebas fue altamente significativa $(\mathrm{p}<0.01)$, pudiéndose constatar la poca utilidad del método visual indirecto aplicado al recuento de garrapatas ingurgitadas en fase parásita, no así del método palpatorio.

Palabras clave: ectoparásitos, bovinos, método palpatorio, método visual indirecto

\section{Abstract}

The study aimed to compare the accuracy of tests or methods for counting engorged ticks in cows reared under extensive production systems. In total, 49 adult cows of the Criollo Neembucú, Brown Swiss, Brangus and Nelore genotypes were used during the summer in March 2019. Several body regions were reviewed sequentially using the indirect visual method, palpatory and finally the direct method, defined the latter as the gold standard. To estimate the validity and reliability indicators, comparison of the behavior of the receiver operating characteristics curves (ROC) and concordance tests, the Epidat 3.1 program for epidemiological analysis of tabulated data was used. The sensitivity and negative predictive value were markedly higher for the palpatory test. The poor value of the Kappa index revealed that the agreement between the indirect and direct visual method was due to random ( $\mathrm{p}=0.2097)$. In the palpatory method, the ROC curve showed a larger area compared to the indirect visual method. The results of the homogeneity test of the ROC areas revealed that the difference in the efficacy of both tests was highly significant $(p<0.01)$, proving the limited usefulness of the indirect visual method applied to the count of engorged ticks in the parasitic phase, as opposed to the palpatory method.

Key words: ectoparasites, bovines, palpatory method, indirect visual method

\section{INTRODUCCIÓN}

Las garrapatas son ácaros competentes para parasitar toda clase de vertebrados, excepto peces; tienen un marcado dimorfismo sexual y hábitos estrictamente hematófagos, exhiben un complejo ciclo de vida caracterizado por una fase parásita de alimentación sanguínea y una fase de vida libre en la cual efectúan la ovoposición y las mudas (Faccioli, 2011).

Las garrapatas, dependiendo del género y especie pueden afectar a uno, dos o más hospederos y su importancia radica, por un lado, en el impacto económico al afectar la productividad de los sistemas ganaderos, mayores inversiones en insumos y mano de obra (Orihuela y Vázquez-Prats, 2008), y por otro, por ser vectores de enfermedades que impactan en la salud de los animales y representan potenciales amenazas para la salud humana. En ese sentido, si bien solo el 10\% de las especies son consideradas de importancia clínico-veterinaria por la epidemiología de ciertas enfermedades zoonóticas, muchas de ellas están implicadas en el mantenimiento de microorganismos, patógenos o no, en el medio ambiente (Faccioli, 2011).

En Paraguay se han reportado varias especies categorizadas como endémicas, tales como Amblyomma aureolatum, A. auricularium, A. brasiliense, A. cajennense, A. dubitatum, A. parvum, A. tigrinum, Dermacentor nitens y Rhipicephalus microplus, esta última ampliamente distribuida 
en la región oriental, y capaces de parasitar mamíferos domésticos de importancia zootécnica, así como representantes de la fauna silvestre, de relevancia por su influjo en la dinámica epidemiológica (Nava et al., 2007).

Por otro lado, la producción animal debe tomar en cuenta la importancia de la sostenibilidad económica y ecológica, con bajos riesgos ambientales y el uso racional del control químico en infestaciones por ectoparásitos, a fin de prevenir la resistencia a los mismos y disminuir los costos ambientales (Saueressig, 2007). En ese sentido, se ha propuesto el manejo para el control de plagas (Fernández, 2009; Cantú y García, 2013), que consiste en la aplicación de varias tecnologías de manera racional, programada y estratégica, requiriendo para el efecto de información generada con rigor científico y adecuada interpretación de los resultados.

Siguiendo la misma línea de razonamiento, varios autores (De la Vega et al., 2009; Cantú y García, 2013) han propuesto un umbral o límite máximo de garrapatas suficiente para mantener la estabilidad enzoótica del rebaño. Este número de garrapatas presente en el animal varía de acuerdo con la dinámica poblacional de las especies involucradas, las características ambientales y climáticas, y la resistencia o susceptibilidad de los hospederos, entre otros aspectos. Con el fin de justificar el establecimiento de un tratamiento de soporte dentro de un programa de control junto a la planificación de medidas estratégicas, se han propuesto métodos para el recuento de ectoparásitos que incluyen principalmente el visual indirecto (Mariscal y Moreno, 2013), el palpatorio y el visual directo (Cantú y García, 2013).

En ese contexto, la presente investigación tuvo como objetivo comparar la exactitud de las pruebas o métodos de recuento de garrapatas ingurgitadas en hembras bovinas criadas bajo sistemas de producción extensiva, a fin de comprobar si los resultados obte- nidos son equivalentes en la determinación del número de garrapatas en fase de vida parásita.

\section{Materiales y Métodos}

La investigación se desarrolló en dos establecimientos ganaderos dedicados a la cría y recría de ganado para la producción de terneros, ubicados en el distrito de Humaitá, departamento de Ñeembucú, y en el distrito de Caapucú, departamento de Paraguarí, ambos en la región oriental del Paraguay, ecorregión Chaco húmedo (Bartrina, 2007).

El tipo climático de Thornthwaite corresponde al subhúmedo húmedo, megatérmico y con precipitación pluvial entre $1400 \mathrm{y}$ $1600 \mathrm{~mm} / \mathrm{año}$. El grupo de animales evaluado estuvo constituido por 49 vacas adultas Criollo Neembucú (CRI), Pardo Suizo (PS), Brangus (BG) y Nelore (NE), bajo sistema de producción extensivo, en praderas constituidas por pastizales y en algunos casos, potreros de gramíneas implantadas como Paspalum sp, Cynodon sp y Brachiaria sp. El trabajo de campo fue realizado durante el estío, en marzo de 2019, treinta días posteriores a la aplicación de benzoilurea, método químico implementado en los establecimientos para el control de garrapatas.

Antes del inicio del estudio se realizaron prácticas de entrenamiento tendientes a evitar el error sistemático y aumentar la precisión (reproducibilidad intra-observador) y exactitud de las mediciones. Para identificar el número de garrapatas ingurgitadas iguales o mayores a $4.5 \mathrm{~mm}$ (Wharton y Utech, 1970) se revisó el lado izquierdo de los animales (Gonzalez-Ceron et al., 2009a) utilizando los métodos visual indirecto, palpatorio y visual directo de forma secuencial en cada animal. Cada una de las pruebas fue llevada a cabo por diferentes miembros del equipo de investigación y se mantuvo el enmascaramiento para los responsables de la interpretación de los resultados (Hulley et al., 2007). 
Los animales fueron llevados al corral de aparte y colocados en el brete. Allí se inició la evaluación empleando el método visual indirecto, que consiste en la captura de imágenes fotográficas y el recuento sobre las mismas de garrapatas que denoten estar ingurgitadas. Para el efecto se fotografiaron las regiones de la cabeza, cuello, axila, vientre, ingle, flanco y perineal, todas del lado izquierdo, considerando la nitidez, claridad y presencia mínima de pliegues cutáneos que pudiesen obstaculizar la visualización de teleoginas. Una vez aprobadas, se procedió al registro asentando el número de identificación animal, genotipo, región anatómica y número de fotografía para su posterior procesamiento mediante un programa amplificador de imágenes, trazando líneas imaginarias sobre la superficie corporal de los animales a fin de delimitar las regiones anatómicas de interés y registrando el número de garrapatas ingurgitadas reconocidas como tales (Mariscal y Moreno, 2013).

Para el recuento palpatorio manual se procedió a pasar la palma de la mano a contra pelo por la superficie de cada región corporal, siguiendo el orden descrito en el párrafo previo. Se registró el número de garrapatas que a la palpación eran detectadas como teleoginas, así como el número de identificación animal, genotipo y región anatómica (Cantú y García, 2013).

Finalmente, se aplicó el método visual directo, que permite realizar el recuento de las garrapatas que presenten una dimensión igual o superior a $4.5 \mathrm{~mm}$ de longitud. Para tal efecto, todas las garrapatas presentes en las regiones evaluadas fueron retiradas e identificadas taxonómicamente como Rhipicephalus (Boophilus) microplus, conocida vulgarmente como la garrapata común del bovino. Las teleoginas se midieron utilizando un vernier digital, con rango de medición de $150 \mathrm{~mm}$ y precisión de $0.01 \mathrm{~mm}$, consignando el número de identificación animal, genotipo, región anatómica, cantidad y dimensión de las garrapatas (Fernández, 2009).
Para la visualización descriptiva de los datos se utilizó el software R (R Core Team, 2020). A fin de evaluar la exactitud de los métodos visual indirecto y palpatorio se determinó un patrón de referencia o gold standard (Hulley et al., 2007) constituido por el método visual directo. Para esto, se consideraron animales positivos o afectados por garrapatas aquellos con número igual o superior a 10 teleoginas por lado, debido a que cantidades por debajo del límite propuesto son adecuadas para mantener la estabilidad enzoótica del rebaño (De la Vega et al., 2009).

Los indicadores de validez y fiabilidad considerados fueron: la sensibilidad, estimada en base a la razón entre animales afectados y el total de positivos; la especificidad, como el cociente entre animales sanos y el total de negativos; el índice de validez representado por la razón entre individuos clasificados correctamente y el total de animales; los valores predictivos positivos y negativos, como el cociente entre el número de verdaderos positivos/negativos y el total de animales con resultado positivo/negativo, respectivamente; la razón de verosimilitud positiva, como la razón entre la fracción de verdaderos positivos (sensibilidad) y la fracción de falsos positivos (1-especificidad), mientras que, la razón de verosimilitud negativa, como el cociente entre la fracción de falsos negati$\operatorname{vos}$ (1-sensibilidad) y la fracción de verdaderos negativos (especificidad); el índice de Youden fue estimado en base a la diferencia entre la tasa de verdaderos positivos y la de falsos positivos (Hervada et al., 2004; Hulley et al., 2007).

Para la determinación de los valores de las pruebas diagnósticas simples, comparación de curvas ROC y pruebas de concordancia se recurrió al programa para análisis epidemiológico de datos tabulados Epidat 3.1 (Hervada et al., 2004). 
Cuadro 1. Número de bovinos clasificados como positivos y negativos a la infestación por garrapatas en fase parásita, evaluado mediante tres metodologías de recuento

\begin{tabular}{llcc}
\hline Metodología & Resultado & FA & FR \% \\
\hline Directo & Negativo & 32 & 65 \\
& Positivo & 17 & 35 \\
Visual indirecto & Negativo & 45 & 92 \\
& Positivo & 4 & 8 \\
Palpatorio & Negativo & 30 & 61 \\
& Positivo & 19 & 39 \\
\hline
\end{tabular}

$F A=$ frecuencia absoluta; $F R=$ frecuencia relativa

\section{Resultados y Discusión}

El Cuadro 1 resume el número de animales clasificados como positivos y negativos tras ser evaluados secuencialmente mediante los tres métodos diagnósticos. En ese sentido, animales con cantidades iguales o superiores a 10 teleoginas en el lado izquierdo fueron considerados positivos, mientras que, aquellos con número inferior negativos. De otra parte, la proporción de animales positivos fue baja al utilizar el método visual indirecto (8\%), en comparación con los resultados obtenidos por los métodos palpatorio y visual directo (39 y $35 \%$, respectivamente).

En general, el bajo nivel de afección en los animales evaluados pudo estar relacionado con las características abióticas vinculadas a la región donde se realizó el estudio. Al respecto, es importante puntualizar que en la región del Chaco húmedo los suelos permanecen inundados la mayor parte del año (humedales del Ñeembucú y áreas de influencia) y la cobertura vegetal constituida mayoritariamente por pastizales puede ser escasa, afectando así el desarrollo de huevos y la longevidad de larvas (Guglielmone y Nava, 2013). Por otro lado, la reducción observada en el número de garrapatas ingurgitadas en este trabajo coincide con lo publicado por Gonzalez-Ceron et al. (2009a) quienes señalaron este comportamiento en coincidencia con la reducción de la precipitación pluvial y el aumento de la temperatura

La Figura 1A muestra la distribución de las observaciones aplicando las tres metodologías de recuento. Se destaca la asimetría positiva y una mayor dispersión de los resultados obtenidos a través de los métodos palpatorio y directo, no así con el método visual indirecto donde la variabilidad registrada fue menor. De igual manera, se observaron valores atípicos próximos al valor máximo, lo que podría deberse a la influencia de factores abióticos y bióticos, entre ellos el manejo y la presencia de animales susceptibles (WingChing, 2015), sobre todo en genotipos con mayor proporción de genes Bos taurus (Figura 1B), en congruencia con lo publicado por Guglielmone y Nava (2013).

Los resultados de los indicadores de validez y fiabilidad obtenidos tras la aplicación de los métodos palpatorio e indirecto se presentan en el Cuadro 2. La proporción de animales afectados, en los que el método visual indirecto fue capaz de arrojar resultados 
A

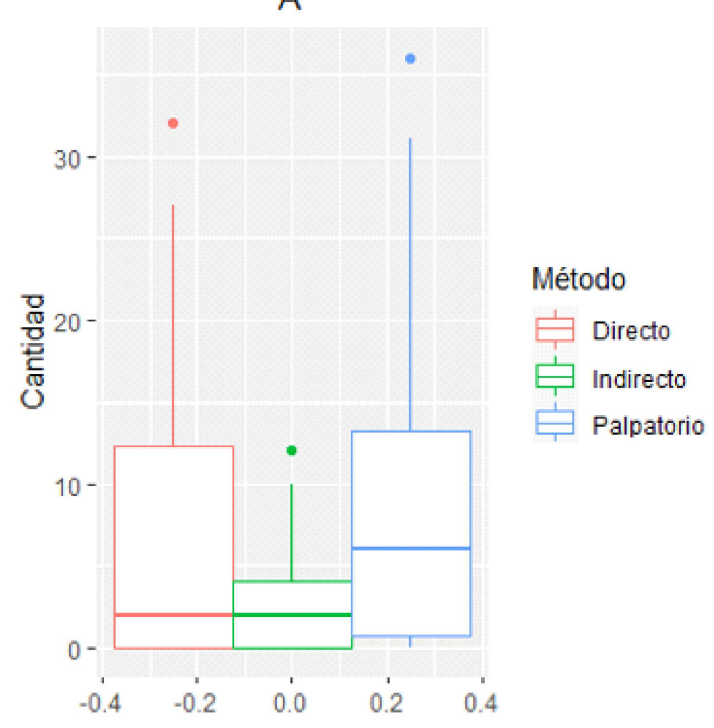

B

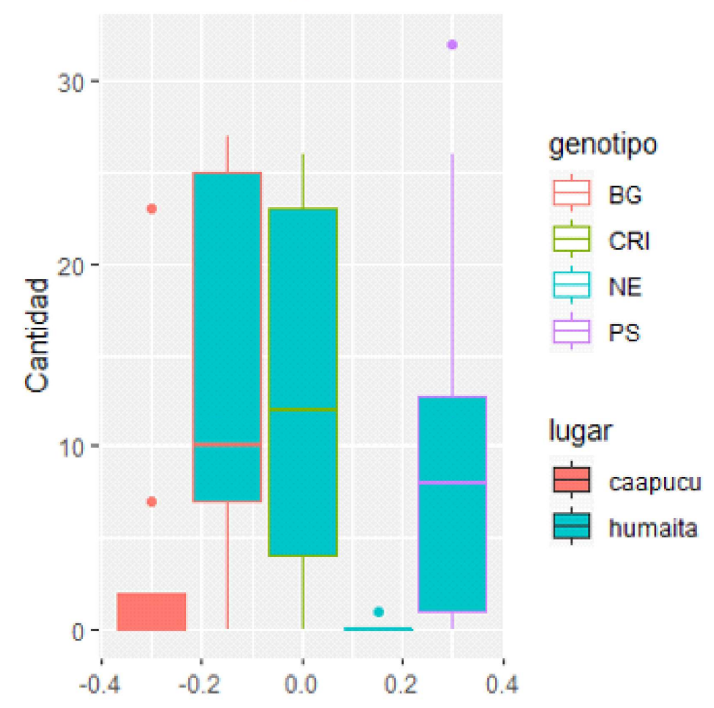

Figura 1. (A) Distribución del número de garrapatas en bovinos evaluados mediante tres metodologías de recuento. (B) Dispersión de las observaciones en bovinos discriminados por genotipo y lugar utilizando el método directo como prueba de referencia o gold standard

positivos, fue marcadamente inferior $(17.65 \%)$ con relación a la sensibilidad registrada con el método palpatorio (100\%).

Por otro lado, la proporción de animales no afectados y definidos como tales fue elevada e idéntica para ambos métodos (93.75\%). En este contexto, es importante sopesar los valores predictivos negativos obtenidos por ambas pruebas; vale decir, la probabilidad condicional de que los individuos con una prueba negativa no cuenten realmente con el umbral mínimo de garrapatas que amerite el establecimiento de medidas alternativas de control, escenario en el que la prueba palpatoria resultó ser más eficaz (100 vs $68.18 \%$ ). A la luz del mismo razonamiento, es importante puntualizar que el valor predictivo positivo fue superior en la prueba palpatoria $(89.47 \%)$ en relación con la visual indirecta $(60 \%)$, lo que equivale a precisar la mayor probabilidad de que un individuo que haya obtenido un resultado positivo en la prueba palpatoria realmente cuente con un número superior a 10 garrapatas en el lado izquierdo al momento de la evaluación.

Otro indicador utilizado para evaluar el desempeño de los métodos fue el índice de Youden, considerado como una medida de eficiencia aplicada a pruebas diagnósticas (Hervada et al., 2004). Este índice fue superior en el método palpatorio (0.94), reflejando de esta manera que la diferencia entre la tasa de verdaderos positivos y la de falsos positivos fue alta; mientras que para el método visual indirecto fue baja (0.11).

La concordancia fue menor entre los métodos visual indirecto y directo (0.67), no así entre el palpatorio y el gold standard. En ese sentido, el valor de acuerdo observado (0.96) mostró mayor coincidencia entre los métodos palpatorio y directo. Paralelamente, 
Cuadro 2. Resumen de los indicadores de validez y fiabilidad de dos métodos de recuento de garrapatas aplicados para la evaluación del nivel de infestación en bovinos

\begin{tabular}{|c|c|c|c|c|}
\hline Indicadores & $\begin{array}{l}\text { Método } \\
\text { indirecto }\end{array}$ & $\mathrm{IC}(95 \%)$ & $\begin{array}{l}\text { Método } \\
\text { palpatorio }\end{array}$ & IC (95\%) \\
\hline Sensibilidad (\%) & 17.65 & $0-38.7$ & 100.00 & $97.1-100$ \\
\hline Especificidad (\%) & 93.75 & $83.8-100$ & 93.75 & $83.8-100$ \\
\hline Índice de validez (\%) & 67.35 & $53.2-81.5$ & 95.92 & $89.4-100$ \\
\hline $\begin{array}{l}\text { Valor predictivo positivo } \\
(\%)\end{array}$ & 60.00 & $7.1-100$ & 89.47 & $73.0-100$ \\
\hline $\begin{array}{l}\text { Valor predictivo negativo } \\
(\%)\end{array}$ & 68.18 & $53.3-83.1$ & 100.00 & $98.3-100$ \\
\hline Prevalencia (\%) & 34.69 & $20.4-49.1$ & 34.69 & $20.4-49.1$ \\
\hline $\begin{array}{l}\text { Razón de verosimilitud } \\
\text { positiva }\end{array}$ & 2.82 & $0.52-15.30$ & 16.00 & $4.18-61.2$ \\
\hline $\begin{array}{l}\text { Razón de verosimilitud } \\
\text { negativa }\end{array}$ & 0.88 & $0.69-1.11$ & - & - \\
\hline Índice de Youden & 0.11 & $-0.09-0.31$ & 0.94 & $0.85-1.02$ \\
\hline
\end{tabular}

Cuadro 3. Análisis de concordancia entre los métodos visual indirecto y palpatorio para determinar el número de garrapatas ingurgitadas en el cuerpo del vacuno con relación a la prueba de referencia (método directo)

\begin{tabular}{ccccccccc}
\hline Métodos & $\begin{array}{c}\text { Acuerdo } \\
\text { observado }\end{array}$ & $\kappa$ & E.E. & $\begin{array}{c}\mathrm{IC} \\
(95 \%)\end{array}$ & $\begin{array}{c}\kappa \\
\mathrm{min}\end{array}$ & $\begin{array}{c}\kappa \\
\text { máx. }\end{array}$ & $\begin{array}{c}\text { Estadístico } \\
\mathrm{Z}\end{array}$ & Valor $\mathrm{p}$ \\
\hline Indirecto & 0.67 & 0.14 & 0.12 & $\begin{array}{c}-0.09- \\
0.37\end{array}$ & -0.19 & 0.41 & 1.25 & 0.2097 \\
Palpatorio & 0.96 & 0.91 & 0.06 & $\begin{array}{c}0.79- \\
1.00\end{array}$ & -0.02 & 0.92 & 6.41 & 0.0000 \\
\hline
\end{tabular}

к = Coeficiente Kappa; $\mathrm{\kappa}$ min= Kappa mínimo; $\mathrm{k}$ máx.= Kappa máximo; E.E.= error estándar; IC= intervalo de confianza 
Cuadro 4. Áreas ROC de los métodos visual indirecto y palpatorio aplicados en el diagnóstico de la infestación por garrapatas en bovinos

\begin{tabular}{lccc}
\hline $\begin{array}{l}\text { Métodos de } \\
\text { diagnóstico }\end{array}$ & Área ROC & E.E. & IC (95\%) \\
\hline $\begin{array}{l}\text { Visual indirecto } \\
\text { Palpatorio }\end{array}$ & 0.5726 & 0.0501 & $0.4743-0.6709$ \\
\hline
\end{tabular}

$\mathrm{ROC}=$ Receiver operating characteristic curve; $\mathrm{E} . \mathrm{E} .=$ error estándar; $\mathrm{IC}=$ intervalo de confianza

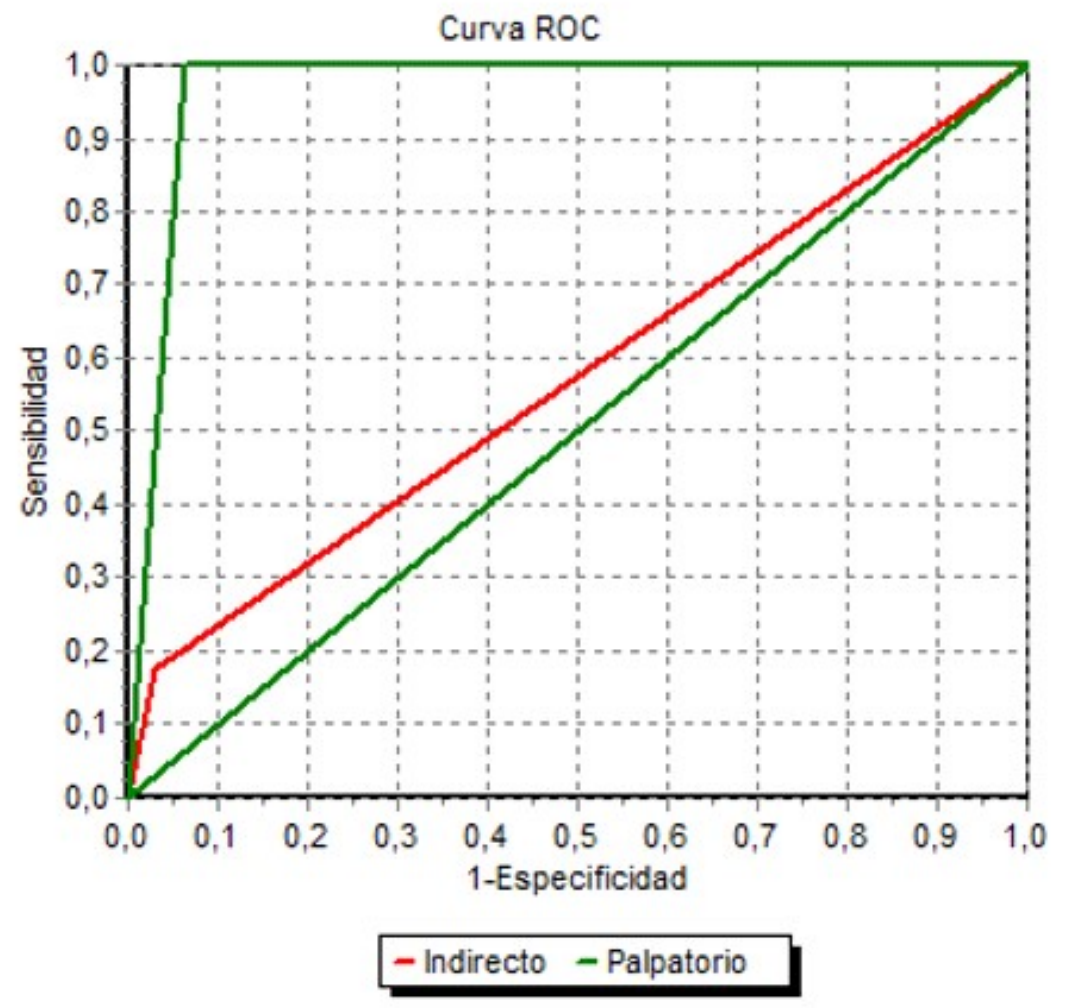

Figura 2. Comparación de curvas ROC (Receiver Operating Characteristic Curve) para el diagnóstico de infestación por garrapatas a través de los métodos visual indirecto y palpatorio 
el índice Kappa (0.91) indicó que la concordancia fue significativamente diferente. En congruencia, el error estándar del estadístico reveló la precisión de la estimación (Hervada et al., 2004; Hulley et al., 2007). Contrariamente el valor deficiente del índice kappa $(0.14)$ obtenido entre los métodos visual indirecto y directo reflejaron que el acuerdo observado entre ambas pruebas fue debido al $\operatorname{azar}(p=0.2097)$. Al respecto, varios autores coinciden en señalar que valores de índices Kappa por encima de 0.75 suelen ser considerados de muy buenos a excelentes (Hervada et al., 2004; Hulley et al., 2007).

En el Cuadro 4 se compara el comportamiento de las curvas de características operacionales del receptor (ROC, por sus siglas en inglés Receiver Operating Characteristic Curve). Los resultados muestran un área mayor para el método palpatorio frente al método visual indirecto $(0.97$ y 0.57 , respectivamente), registrando en ambos casos valores de error estándar bajos que revelaron estimaciones precisas.

En el análisis de comparación de las curvas de eficacia diagnóstica es importante considerar que aquellas que siguen la diagonal arrojan el mismo número de verdaderos y falsos positivos (Hulley et al., 2007), situación que fue verificada en la prueba visual indirecta, pudiéndose constatar de esta manera su poca utilidad con relación al método palpatorio (Figura 2). Esta circunstancia pudo deberse al hecho de que, si bien las fotografías fueron sometidas a criterios de selección, fue inevitable la presencia de algunos pliegues cutáneos que pudieron haber enmascarado la presencia de garrapatas, máxime en las regiones axilar e inguinal que habitualmente son más afectadas (González-Cerón et al., 2009b; WingChing, 2015).

Finalmente, los resultados de la prueba de homogeneidad de las áreas ROC indicaron que la diferencia en cuanto a la eficacia de ambas pruebas fue altamente significativa $(p<0.01)$.

\section{Conclusiones}

- Existe concordancia entre los métodos palpatorio y directo para el recuento de garrapatas de fase parásita en animales de la especie bovina.

- La baja sensibilidad y el acuerdo deficiente entre los métodos visual indirecto y directo revelan la falta de utilidad de la prueba para determinar el número de garrapatas ingurgitadas.

- El método de recuento palpatorio es eficaz a la hora de precisar el número de teleoginas.

\section{Agradecimientos}

A los productores, Ing. Agr. Guillermo Souto y MV Gustavo Granada, quienes gentilmente acompañaron e hicieron posible este trabajo. El proyecto fue financiado por el CONAYT a través del programa PROCIENCIA con recursos del Fondo para la Excelencia de la Educación e Investigación - FEEI. La presente publicación ha sido elaborada con el apoyo del CONACYT. Su contenido es responsabilidad exclusiva de los autores y en ningún caso se debe considerar que refleja la opinión del CONACYT.

\section{Literatura Citada}

1. Bartrina L. 2007. Contexto geográfico general. En: Biodiversidad del Paraguay: una aproximación a sus realidades. Asunción: Fundación Moisés Bertoni. p 25-32.

2. Cantú A, García Z. 2013. Estrategias para el control integrado de garrapata (Boophilus spp) en la producción de bovinos de carne en pastoreo. México: INIFAP. $38 \mathrm{p}$.

3. De la Vega R, Díaz G, García I, Camejo A. 2009. Application of tick sampling on acaricidal bath frequency in Rhipicephalus (Boophilus) microplus (Acari: Ixodidae). Rev Salud Anim 31: 105-107. 
4. Faccioli V. 2011. Serie de Catálogos. Garrapatas (Acari: Ixodidae y Argasidae) de la colección de invertebrados del Museo Provincial de Ciencias Naturales» Florentino Ameguino». Serie de Catálogos $\mathrm{N}^{\circ} 25$. Santa Fé: Santa Fe. Argentina. $34 \mathrm{p}$.

5. Fernández M. 2009. Manejo integrado de Boophilus microplus. En: Perspectivas de control biológico parasitario y nuevas alternativas en el sector pecuario. México: CENID-PAVET-INFAP. $p$ 227-267.

6. González-Cerón F, Becerril-Pérez CM, Torres-Hernández G, Díaz-Rivera P. 2009a. Garrapatas que infestan regiones corporales del bovino Criollo Lechero Tropical en Veracruz, México. Agrociencia 43: 11-19.

7. Gonzalez-Ceron F, Becerril-Perez CM, Torres-Hernandez G, Diaz-Rivera P, Santellano-Estrada E, RosendoPonce A. 2009b. Infestación natural por Amblyomma cajennense y Boophilus microplus en bovinos Criollo Lechero Tropicial durante la época de lluvias. Agrociencia 43: 557-584.

8. Guglielmone AA, Nava S. 2013. Epidemiologia y control de las garrapatas de los bovinos en la Argentina. En: Enfermedades parasitarias con importancia clínica y productiva en rumiantes: fundamentos epidemiológicos para su diagnóstico y control . Buenos Aires: Hemisferio Sur. p 441-456.

9. Hervada X, Pérez MI, Vázquez E, Castillo C, Loyola E, Ayçaguer LC. 2004. Epidat: Programa para análisis epidemiológico de datos tabulados. v. 3.1. Rev Española Salud Pública 78(2). doi: 10.1590/S1135-57272004000200013

10. Hulley SB, Cummings SM, Browner WS, Grady DG, Newman TB. 2007. Diseño de investigaciones clínicas. Philadelphia, USA: Lippincott Williams \&Wilkins. 417 p.
11. Mariscal P, Moreno J. 2013. Prevalencia de Haematobia irritans (Linnaeus 1758) (Díptera: Muscidae) en bovinos de la Provincia Cercado, Beni. Agrociencias Amaz 1: 31-42.

12. Nava S, Lareschi M, Rebollo $C$, Benitez Usher C, Beati L, Robbins RG, Durden LA, Mangold AJ, Guglielmone AA. 2007. The ticks (Acari: Ixodida: Argasidae, Ixodidae) of Paraguay. Ann Trop Med Parasitol 101: 255270. doi: 10.1179/136485907X176319

13. Orihuela A, Vázquez-Prats VM. 2008. Behavioral strategies in host-parasite relationships. Tec Pecu Mex 46: 259-285.

14. $R$ Core Team. 2020. R: A language and environment for statistical computing. $\mathrm{R}$ Foundation for Statistical Computing. Vienna, Austria. URL https://www.Rproject.org/

15. Salinas E, Falcón N. 2020. Concordancia entre el diagnóstico presuntivo y tomográfico en canes con enfermedades encefálicas y de columna vertebral. Rev Inv Vet Perú 31: e18167. doi: 10.15381/rivep.v31i3.18167

16. Saueressig T. 2007. Control racional de las parasitosis bovinas con bajo impacto ambiental. En: XI Seminario Manejo y Utilización de Pastos y Forrajes en Sistemas de Producción Animal. Barquisimeto: Funda Pastos.

17. Wharton R, Utech K. 1970. The relation between engorgement and dropping of Boophilus micropus (Canestrini) (Ixodidae) to the assessment of tick numbers on cattle. Aust J Entomol 9: 171-182. doi: 10.1111/j.1440-6055.1970.tb00788.x

18. WingChing JR. 2015. Extracción manual de garrapatas Riphicephalus (Boophilus) microplus en ganado bovino como estrategia de control. Nutr Anim Trop 9: 88. doi: 10.15517/NAT.V9I1.19393 